\title{
CCL2 promotes macrophages-associated chemoresistance via MCPIP1 dual catalytic activities in multiple myeloma
}

\author{
Ruyi Xu', Yi Li', Haimeng Yan'1, Enfan Zhang ${ }^{1}$, Xi Huang ${ }^{1}$, Qingxiao Chen'1, Jing Chen ${ }^{1}$, Jianwei Qu', Yang Liu', \\ Jingsong $\mathrm{He}^{1}$, Qing $\mathrm{Yi}^{2}$ and Zhen Cai $\mathbb{C}^{1}$
}

\begin{abstract}
We previously showed that the chemokine CCL2 can recruit macrophages (M $\varphi s)$ to the bone marrow (BM) in multiple myeloma (MM) and that myeloma-associated $M \varphi s$ are important in drug resistance. Here, we explore the role of increased CCL2 expression in the BM microenvironment of MM and elucidate the underlying mechanism. Our results show that CCL2 expression is associated with the treatment status of MM patients. M $\varphi s$ interact with MM cells and further upregulate their expression of CCL2. These increased level of CCL2 polarizes M 9 s toward the M2-like phenotype and promotes M $\varphi s$ to protect MM cells from drug-induced apoptosis. Mechanistically, CCL2 upregulated

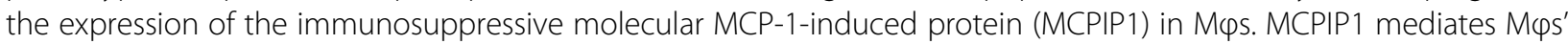
polarization and protection via dual catalytic activities. Additionally, we found that CCL2 induces MCPIP1 expression via the JAK2-STAT3 signaling pathway. Taken together, our results indicate that increased CCL2 expression in MM

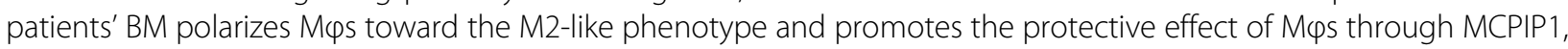
providing novel insight into the mechanism of Mys-mediated drug resistance in MM.
\end{abstract}

\section{Introduction}

Multiple myeloma (MM) is an incurable hematologic malignancy characterized by the accumulation of monoclonal plasma cells in the bone marrow $(\mathrm{BM})^{1}$. Despite the introduction of novel chemotherapy agents, chemoresistance remains the major problem in clinical management of $\mathrm{MM}^{2}$.

Regardless, the mechanism of MM chemoresistance has not yet been fully elucidated. Some studies have shown that MM cells possess clonal heterogeneity, and such mutations may result in resistance to chemotherapy ${ }^{3}$. In addition, other studies have reported that the BM plays an essential role in $\mathrm{MM}$ chemoresistance ${ }^{4,5}$, and that the

\footnotetext{
Correspondence: Zhen Cai (caiz@zju.edu.cn)

${ }^{1}$ Bone Marrow Transplantation Center, The First Affiliated Hospital, School of Medicine, Zhejiang University, Hangzhou, China

${ }^{2}$ Center for Hematologic Malignancy, Research Institute, Houston Methodist, Houston, TX, USA

These authors contributed equally: Ruyi Xu, Yi Li

Edited by J.-E. Ricci
}

interaction of MM cells with different cell components of the tumor microenvironment is important for tumor growth and chemoresistance ${ }^{6}$.

Macrophages $(\mathrm{M} \phi \mathrm{s})$ are prominent components in the $\mathrm{BM}$ microenvironment of $\mathrm{MM}^{7,8}$. We previously found that $M \phi s$ protect MM cells from drug-induced apoptosis ${ }^{7}$. In addition, $M \phi s$ provide a favorable microenvironment for MM cells via crosstalk with other stromal cells and may also promote $\mathrm{MM}$ chemoresistance through $\mathrm{M} \phi \mathrm{s}-$ MM cells interaction ${ }^{8}$. M $\phi s$ possess great plasticity and can differentiate into different functional states according to microenvironmental signals ${ }^{9}$. M $\phi s$ can be classified into two major polarized states: M1-M $\phi \mathrm{s}$, which have remarkable tumoricidal activity; and $\mathrm{M} 2-\mathrm{M} \phi \mathrm{s}$, which generally suppress antitumor immunity ${ }^{10}$. Many in vivo studies have indicated that tumor-associated M (TAMs) are often educated to develop M2-like phenotypes in advanced stages of cancer ${ }^{11,12}$. Nonetheless, how $\mathrm{M} \phi \mathrm{s}$ are polarized toward the M2-like phenotype and

\section{(c) The Author(s) 2019}

(c) (i) Open Access This article is licensed under a Creative Commons Attribution 4.0 International License, which permits use, sharing, adaptation, distribution and reproduction in any medium or format, as long as you give appropriate credit to the original author(s) and the source, provide a link to the Creative Commons license, and indicate if changes were made. The images or other third party material in this article are included in the article's Creative Commons license, unless indicated otherwise in a credit line to the material. If material is not included in the article's Creative Commons license and your intended use is not permitted by statutory regulation or exceeds the permitted use, you will need to obtain permission directly from the copyright holder. To view a copy of this license, visit http://creativecommons.org/licenses/by/4.0/. 
whether the M2-like phenotype is associated with the protective effect of $\mathrm{M} \phi$ s have not yet been fully defined.

Our previous study showed that the chemokine CCL2 promoted M $\phi s^{\prime}$ infiltration in the MM-BM microenvironment and encouraged $M \phi s^{\prime}$ proliferation ${ }^{13}$. CCL2, also known as MCP-1, is a member of the CC family of chemokines with an affinity for the receptor CCR $2^{14}$. CCL2 has been shown to be a critical modulator of inflammation and recruitment of monocytes, NK cells, and $\mathrm{T}$-cell subpopulations in numerous diseases ${ }^{15,16}$. In addition to regulating immune cell migration, CCL2 contributes to increased angiogenesis and bone resorption, two clinical features often observed in $\mathrm{MM}^{17,18}$. Some studies have shown that CCL2 plays a role in educating M $\phi s$ to become M2-like $M \phi s^{19}$, but many other studies have indicated that CCL2 is a marker of M1-like $M \phi s^{20}$.

In this study, we aimed to investigate the significance of CCL2 in M $\phi s-$ mediated MM chemoresistance. We performed mechanistic studies to further elucidate how CCL2 regulates M $\phi s^{\prime}$ function with regard to MM cells in vitro and in vivo. Our results provide new insight into the mechanism of drug resistance in MM.

\section{Materials and methods \\ Cells}

Human MM cell lines ARP-1, RPMI-8226, MM.1S, CAG, JJN3, and OPM2 were generously provided by Dr. Qing Yi (Center for Hematologic Malignancy, Research Institute, Houston Methodist, Houston, TX, USA) and cultured in RPMI-1640 medium containing 10\% fetal bovine serum (FBS, Thermo Fisher Scientific, Gibco, Waltham, MA, USA) and $1 \% \mathrm{~L}$-glutamine at $37^{\circ} \mathrm{C}$ in $5 \%$ $\mathrm{CO}_{2}$ in air. Conditioned medium of MM cells was acquired from culture supernatants, which were seeded at $5 \times 10^{5}$ cells $/ \mathrm{mL}$ for $24 \mathrm{~h}$.

$\mathrm{PBMCs}$ (peripheral blood mononuclear cells) were isolated from healthy donors after obtaining informed consent. Human M $\phi s$ were generated from PBMCs as previously described ${ }^{7}$. Briefly, monocytes were incubated in 6-well plates for $1-2 \mathrm{~h}$ at $37^{\circ} \mathrm{C}$; nonadherent cells were removed, and the adherent monocytes were incubated for 5-7 days in medium containing M-CSF $(20 \mathrm{ng} / \mathrm{ml}$; R\&D Systems, MN, USA). Before use, the M $\phi$ s were phenotyped by morphological analysis, and the molecular marker CD14, CD68 were also detected (Supplementary Fig. $4 \mathrm{~A}-\mathrm{C})$.

MM cells were cocultured with $\mathrm{M} \phi \mathrm{s}$ at a 1:1 ratio for $24 \mathrm{~h}$ with bortezomib ( $10 \mathrm{nM}$, Selleckchem, TX, USA) or melphalan $(15 \mu \mathrm{M}$, MedChemExpress, NJ, USA). Then suspended MM cells were collected for functional assays to determine the protective effect of $M \phi s$ on bortezomib/ melphalan-induced MM cell apoptosis. In some experiments, MM cells were cultured in Transwell inserts (0.4 $\mu \mathrm{m}$ pore size, Corning Inc., Tewksbury, MA, USA).
For inhibition experiments, M $\phi$ s were preincubated with Stattic (MedChemExpress, NJ, USA) for $2 \mathrm{~h}$ before rhCCL2 (50 ng/ml, R\&D System, MN, USA) treatment. A neutralizing anti-CCL2 antibody $(\alpha C C L 2,50 \mu \mathrm{g} / \mathrm{ml})$ was purchased from R\&D Systems, MN, USA.

\section{Transient siRNA transfection}

A scrambled nontargeting siRNA and one siRNA targeting MCPIP1 (MCPIP1-Homo-1694:5'GGUCUGAAC CAUACCCACUTT-3') were obtained from GenePharm, Shanghai, China. The siRNAs were transfected into cells according to the manufacturer's protocol. Briefly, attached M $\phi s$ were incubated in Opti-MEM (catalog number 11058-021, Invitrogen, Carlsbad, CA, USA) with a complex of MCPIP1 siRNA or control siRNA and Lipofectamine 2000 transfection reagent (catalog number 11668027, Invitrogen, Carlsbad, CA, USA) for $24 \mathrm{~h}$. The medium containing the siRNA transfection reagent complexes was then aspirated and replaced with RPMI1640 medium and 10\% FBS for $24 \mathrm{~h}$ before functional studies.

\section{Lentiviral transfection}

For wild-type MCPIP1 and D141N mutant MCPIP1 overexpression, homo wild-type (WT) MCPIP1 cDNA or homo D141N mutant MCPIP1 cDNA and the GFP gene were inserted into the pCDH-CMV-EF1-T2A-Puro lentiviral vector. Lentiviral vectors were purified and then transfected into 293T cells, and lentiviral particles were collected after $48 \mathrm{~h}$. Attached M $\phi$ s were transduced with LV-WT MCPIP1 or LV-D141N MCPIP1 (multiplicity of infection: 50) lentiviral vectors in the presence of polybrene $(5-10 \mu \mathrm{g} / \mathrm{mL})$ for $12 \mathrm{~h}$. The empty vector (pCDHCMV-EF1-T2A-Puro) was used as the negative control. MCPIP1 protein expression in the cells was examined by Western blotting after viral infection for $48 \mathrm{~h}$.

\section{Quantitative real-time PCR}

Total mRNA was isolated from cells using RNAiso ${ }^{\mathrm{TM}}$ PLUS (TaKaRa, Shiga, Japan), and cDNA synthesis was performed using a PrimeScript ${ }^{\text {TM }}$ RT Reagent Kit with DNA Eraser (TaKaRa, Shiga, Japan). Quantitative realtime polymerase chain reaction (qRT-PCR) was carried out with SYBR Premix Ex Taq II (TiRNaseH Plus) (Takara, Shiga, Japan) and a Bio-Rad CFX96 real-time system (Bio-Rad, Hercules, CA, USA) according to the manufacturer's instructions. Data were analyzed using the relative standard curve method and normalized to GAPDH. The primer sets used for these analyses are summarized in Supplementary Table 1.

\section{Western blot analysis}

Cells were collected and extracted with lysis buffer containing a protease and phosphatase inhibitor cocktail 
(Thermo Fisher Scientific, Waltham, MA, USA). Equal amounts of protein were separated by sodium dodecyl sulfate polyacrylamide gel electrophoresis and then transferred onto polyvinylidene difluoride membranes (Merck Millipore, Darmstadt, Germany). The membranes were blocked with 5\% nonfat milk for $1-2 \mathrm{~h}$ and then incubated with corresponding primary antibodies overnight at $4{ }^{\circ} \mathrm{C}$. The membranes were washed with Trisbuffered saline with Tween 20 (TBST) and incubated with an horseradish peroxidase (HRP)-conjugated anti-rabbit or anti-mouse antibody in TBST for $1 \mathrm{~h}$ at room temperature. The membranes were washed three times with TBST, and the protein bands were detected using a ChemiDoc $^{\text {TM }}$ MP Imaging System (Bio-Rad) and an enhanced chemiluminescence detection kit (Biological Industries, Israel, Beit Haemek Ltd., Kibbutz Beit Hamek, Israel). Primary antibodies, including anti-p-AMPK, -caspase-3, -caspase-9, -Bad, -Bcl2, -Bax, -Bcl-xl, -STAT3, -p-STAT3 (Tyr705), -pSTAT3 (Ser727), -JAK2, -p-JAK2 (Y1008), and -SOCS3 were obtained from Cell Signaling Technology (MA, USA). Anti-iNOS and -PARP-1 antibodies were purchased from Abcam (Cambridge, UK). An anti-GAPDH antibody was purchased from SigmaAldrich, Billerica (MA, USA), and an anti-MCPIP1 antibody was purchased from Santa Cruz Biotechnology, CA, USA.

\section{Proteome profiler human phospho-kinase antibody array}

Proteome profiler human phospho-kinase antibody array was utilized according to the manufacturer's protocol. In this array, 46 captured antibodies or control antibodies against human phosphorylated kinases are spotted in duplicate on nitrocellulose membranes.

Before harvesting, $M \phi s$ were treated with PBS or rhCCL2 for $24 \mathrm{~h}$ and lysed using lysis buffer (ARY003, Proteome Profiler ${ }^{\mathrm{TM}}$, R\&D Systems, Minneapolis, MN, USA). The total protein concentration of each sample was quantified using a Bio-Rad DC protein assay kit II (Cat \#500-0112, Bio-Rad Laboratories, Philadelphia), and $150 \mu \mathrm{g} / \mathrm{mL}$ was used for the array. The lysates were incubated overnight with the array membranes, and after $24 \mathrm{~h}$, they were washed to remove any unbound proteins. Further incubation was performed with a cocktail of biotinylated detection antibodies for $2 \mathrm{~h}$ at room temperature. The membranes were then exposed to streptavidin-HRP for $30 \mathrm{~min}$. After a final wash, the proteins bound to the membrane were detected by exposure to an enhanced chemiluminescent reagent for $1 \mathrm{~min}$. Chemiluminescent images were captured using a Syngene G-BOX (G: BOX-CHEMI_XL1.4, Syngene, Cambridge, UK). To quantify activation levels of the proteins, the integrated optical density (IOD) of each spot was assessed at increasing exposure using a Bio-Rad ChemiDoc station; the IOD values were corrected for background signals. To compare different membranes, the values were normalized to those of the positive controls on each membrane, and the protein expression levels were then quantified. The experiment was performed twice to confirm the results obtained.

\section{Flow cytometry}

Apoptosis was detected by staining cells with Annexin V-FITC/propidium iodide (Dojindo, Kumamoto, Japan) according to the manufacturer's instructions. CD14Ab (Biolegend, CA, USA) staining was used to exclude M $\phi \mathrm{s}$ from the analysis.

Expression of active caspase- 3 was measured using a Fluorescein Active Caspase-3 Staining Kit (Invitrogen, Carlsbad, CA, USA). Expression of CD206, CD163, and CD86 was measured by direct immunofluorescence using PE-conjugated CD206 (Biolegend, CA, USA) and CD163 (Biolegend, CA, USA) and APC-conjugated CD86 (Biolegend, CA, USA). An isotype control was used to exclude nonspecific signals.

For intracellular CCL2 staining, cells were stimulated with Leukocyte Activation Cocktail (BD Bioscience, CA, USA) for $4 \mathrm{~h}$ at $37^{\circ} \mathrm{C}$. The cells were then fixed and permeabilized with Fixation Buffer and Intracellular Staining Perm Wash Buffer (Biolegend, CA, USA) according to the manufacturer's instructions. Next, the cells were stained with a PE-mouse anti-human MCP-1 antibody (BD Pharmingen, San Diego, CA, USA). Data were acquired with a FACScan flow cytometer (BD Biosciences, San Diego, CA, USA) and analyzed using Flowjo 7.6.1.

\section{Cell proliferation assay}

A CCK-8 proliferation assay (Dojindo, Kumamoto, Japan) was used to assess MM cell proliferation. A total of $1 \times 10^{4} \mathrm{MM}$ cells/well were seeded in 96-well plates and cultured for the indicated times. The cells were treated with CCK-8 solution $(10 \mu \mathrm{L})$ for another $2 \mathrm{~h}$ at $37^{\circ} \mathrm{C}$, and absorbance was measured at $450 \mathrm{~nm}$ using a microplate reader (Bio-Rad, Model 680).

\section{Enzyme-linked immunosorbent assay}

CCL2 in peripheral serum and cell culture supernatants (kits from Biolegend, CA, USA) and IL-10 and TNF- $\alpha$ secreted by $M \phi s$ (kits from Biolegend, CA, USA) were analyzed by ELISA according to the suppliers' instructions.

\section{RNA sequencing}

RNA sequencing was performed following Illumina mRNA Sequencing Sample Preparation Guide (Ilumina, CA, USA). M $\phi s$ generated from PBMCs of 2 independent healthy donors (M $\phi s-1, M \phi s-2)$ were treated with rhCCL2 $(50 \mathrm{ng} / \mathrm{mL})$ for $24 \mathrm{~h}$. Total RNA was then 
extracted using Redzol reagent (SBS Genetech Inc., Beijing, China) and quantified (NanoDrop spectrophotometer, Thermo Fisher Scientific), with quality control using the Agilent BioAnalyzer 2100 system (Agilent Technologies Inc., Beijing, China). Sequencing cluster generation and sequencing were performed with Genome Analyzer IIx (Illumina) at Ruibo Biotechnology Company (Guangzhou, China).

\section{Immunohistochemistry and immunofluorescence}

Formalin-fixed, paraffin-embedded BM biopsy samples from MM patients were examined by immunohistochemistry to detect expression of CCL2. All 12 cases were histopathologically and clinically diagnosed as new untreated MM, and the patients received four courses of PCD (bortezomib + cyclophosphamide + dexamethasome) therapy at The First Affiliated Hospital, School of Medicine, Zhejiang University from January to May 2017. All patients consented to participate in the study, and the study was approved by the Research Ethics Committee of the First Affiliated Hospital, School of Medicine, Zhejiang University. The degree of immunostaining was evaluated using H-Score. Anti-CD138, anti-cleaved caspase-3, and anti-MCPIP1 antibody immunofluorescence analysis was performed. Image-pro plus 6.0 software (Media Cybernetics, Inc., Rockville, MD, USA) was used for quantification of cleaved caspase- 3 signals. The quantitative value of areal density $=$ IOD (integral optical density)/area (pixels area), presenting positive expression of cleaved caspase- 3 in the captured image. Antibodies against cleaved caspase- 3 and CD138 were obtained from Proteintech Group Inc., IL, USA. An anti-MCPIP1 antibody was purchased from Santa Cruz Biotechnology and an anti-CCL2 antibody from Abcam (Cambridge, UK).

\section{MM xenograft model}

Four-week-old female NSG mice were obtained from the Model Animal Research Center of Nanjing University (Nanjing, China) and housed in the animal facility of Zhejiang University School of Medicine. The Tab of Animal Experimental Ethical Inspection of the First Affiliated Hospital, School of Medicine, Zhejiang University approved the procedures and protocols of all experiments. ARP-1 $\left(5 \times 10^{6}\right)$ cells were injected subcutaneously into the right flanks of the mice. After 7 days, when palpable tumors $(>5 \mathrm{~mm})$ had developed, some of the mice were injected intraperitoneally with bortezomib $(2 \mu \mathrm{g} / \mathrm{mouse}$, every 3 days) for 1 week or with PBS as a control. For some mice, differently treated $\mathrm{M} \phi \mathrm{s}$ were injected into the tumor mass, and some mice received CCX140-B (MedChemExpress, NJ, USA) by oral gavage $(500 \mu \mathrm{g} /$ mouse/day) every day for 1 week. Tumor size was monitored daily with calipers, and upon sacrifice, CD14+ cells were purified from the tumors using anti-CD14-coated magnetic microbeads (Miltenyi Biotec, Bergisch Glabach, Germany). These cells were used for RNA extraction.

\section{Statistical analysis}

GraphPad Prism 6 and Excel were used for all statistical analyses. Values represent the means \pm SD for at least three independent experiments performed in triplicate. Significant differences between experimental groups were determined using a two-tailed Student's $t$ test and oneway analysis of variance where appropriate. All $P$ values $<$ 0.05 were considered statistically significant. The significance of $P$ values is ${ }^{*} P<0.05$; ${ }^{* *} P<0.01$, ${ }^{* * *} P<0.001$, NS : not significant.

\section{Results}

\section{Clinical significance of CCL2 expression in MM}

We first examined CCL2 expression in peripheral serum from newly diagnosed MM patients and healthy donors using ELISA. As shown in Fig. 1a, CCL2 could be detected in samples from all patients and donors, with a much higher concentration in the former. Interestingly, we found that after the newly diagnosed patients received four courses of PCD combined therapy, CCL2 expression in their BM was significantly decreased (Fig. 1b, c). Based on the clinicopathologic characteristics we collected, almost all of these patients were in remission as a result of PCD combined therapy (Table 1). These results strongly suggest that CCL2 expression may be tightly associated with the treatment status of MM patients.

\section{MM cells induced CCL2 expression in M $\varphi s$}

We evaluated CCL2 expression in human MM cell lines, M $\phi s$ and PBMCs. qRT-PCR, ELISA, and flow cytometry results all revealed that $\mathrm{MM}$ cell lines and PBMCs barely expressed CCL2 but that M $\phi$ s highly expressed CCL2 (Fig. 1 d, e and Supplementary Fig. 1A). We also identified the expression of CCL2 in CD68+ M $9 \mathrm{~s}$ and CD138+ MM cells from MM patients' BM biopsies using immunofluorescence (Supplementary Fig. 1b), showing CCL2 was mainly expressed by M $\phi$ s. To determine whether MM cells affect CCL2 expression in M $\phi s$, we cocultured $M \phi s$ with MM cells directly or through Transwell chambers for $24 \mathrm{~h}$. According to qRT-PCR, ELISA, and flow cytometry, MM cells significantly induced CCL2 expression in M $\phi s$ (Fig. 1f, g and Supplementary Fig. 1C).

\section{CCL2 did not affect the proliferation and drug responses of MM cells}

To determine the effect of increased CCL2 levels in the $\mathrm{BM}$ microenvironment in MM, we conducted CCK-8 assays to examine whether CCL2 promotes MM cells proliferation. As shown in Supplementary Fig. 1D, there was no difference between groups treated or not with 
A

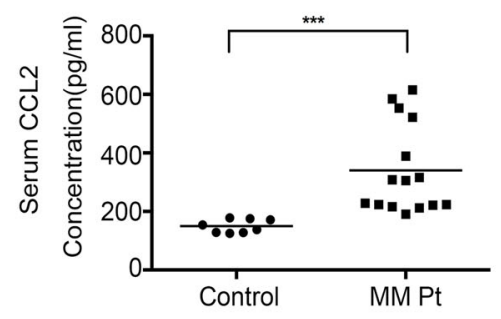

C

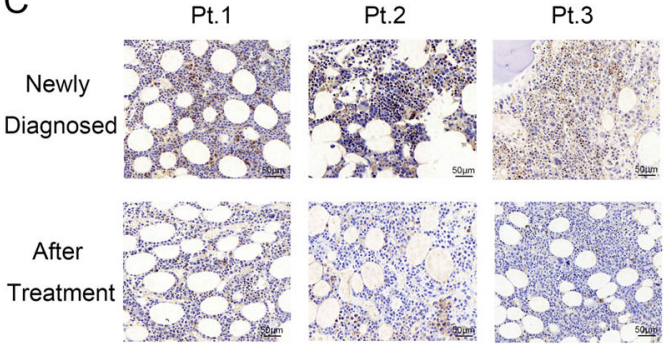

E

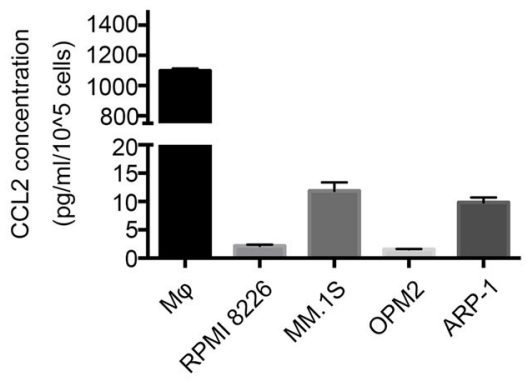

G

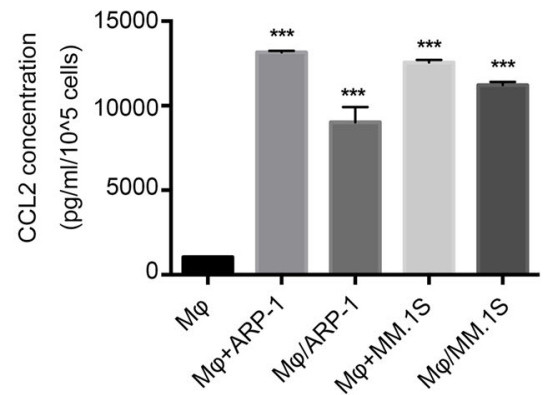

B

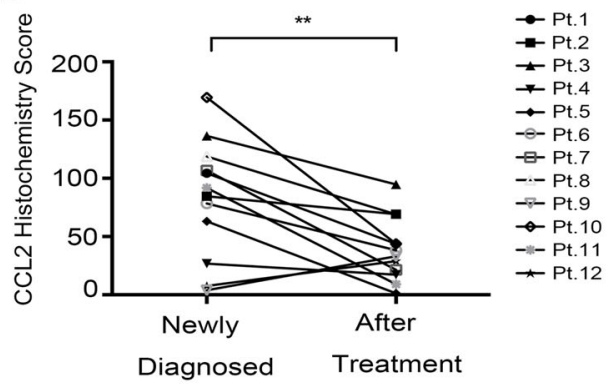

D

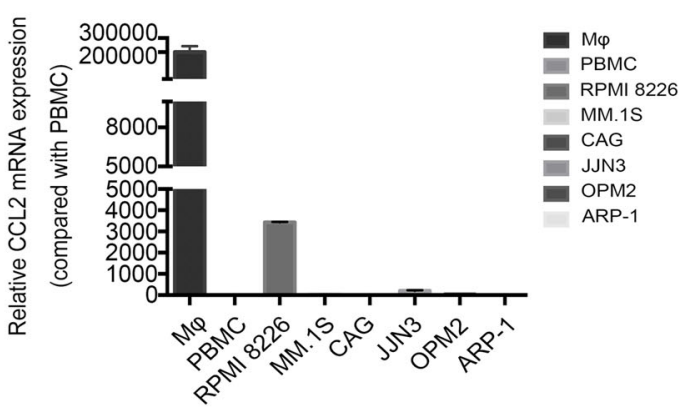

F

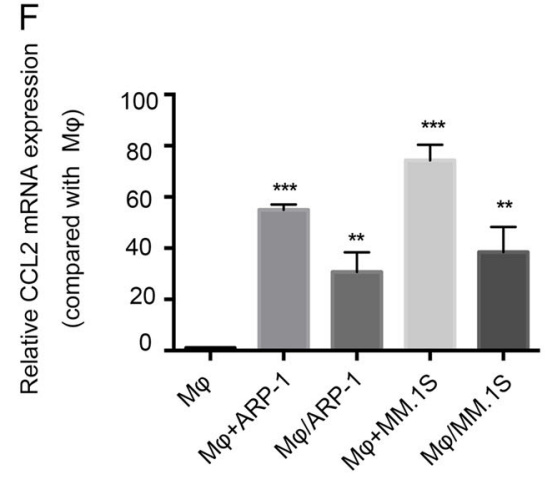

Fig. 1 Clinical significance and expression of CCL2 in MM. a Levels of CCL2, as measured by ELISA, in the peripheral serum of healthy donors (control) and MM patients (MM Pt). Samples from 8 and 15 healthy donors and MM patients, respectively, were used. $\mathbf{b}$ The histochemistry score for CCL2 was detected using BM biopsy samples when the patients were newly diagnosed or after treatment (median H-score from 88.104320 to 29.8143). Pt.1 to Pt.12 represent 12 independent patients. c Immunohistochemical analysis of CCL2 expression in 3 representative BM biopsies (Pt.1 to Pt.3). The top panel shows CCL2 expression when patients were newly diagnosed, and the bottom panel shows CCL2 expression after the same patient received therapy. Scale bars, $50 \mu \mathrm{m}$. d Ratio of mRNA expression of CCL2 in MM cell lines (RPMI 8226, MM.1 S, CAG, JJN3, OPM2, ARP-1), PBMCs and macrophages (M $\varphi s$ ) by RT-PCR. e A total of $1 \times 10^{5}$ cells were cultured in $1 \mathrm{~mL}$ of medium for $24 \mathrm{~h}$, followed by ELISA analysis of CCL2

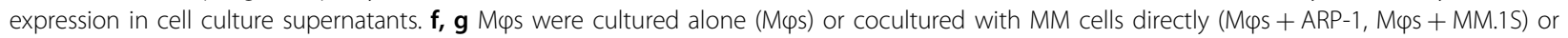

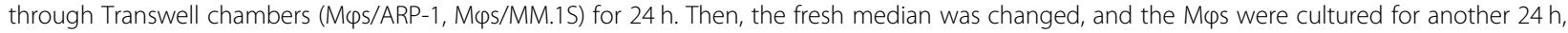
followed by RT-PCR $\mathbf{f}$ and ELISA $\mathbf{g}$ to detect CCL2 expression. Summarized data from at least three independent experiments are shown. Values are presented as means $\pm \mathrm{SD}$. ${ }^{*} P<0.05 ;{ }^{*} P<0.01 ;{ }^{* *} P<0.001$ 
Table 1 Patient characteristics

\begin{tabular}{|c|c|c|c|}
\hline Variables & $\begin{array}{l}\text { Newly diagnosed } \\
(n=12)\end{array}$ & $\begin{array}{l}\text { After PCD } \\
\text { treatment } \\
(n=12)\end{array}$ & $P$ \\
\hline Median age, years (range) & $59(39-74)$ & & \\
\hline $\operatorname{Sex}(M / F)$ & $4 / 8$ & & \\
\hline \multicolumn{4}{|l|}{$\begin{array}{l}\text { Durie-Salmon staging, } n \\
(\%)\end{array}$} \\
\hline Stage IA & - & $3(25 \%)$ & \\
\hline Stage IIA & $1(8.33 \%)$ & - & \\
\hline Stage IIB & - & - & \\
\hline Stage IIIA & $9(75 \%)$ & $8(66.67 \%)$ & \\
\hline Stage IIIB & $2(16.67 \%)$ & $1(8.33 \%)$ & \\
\hline \multicolumn{4}{|l|}{ ISS, n (\%) } \\
\hline Stage I & - & - & \\
\hline Stage ॥ & $7(58.33 \%)$ & $12(100 \%)$ & \\
\hline Stage III & $5(41.67 \%)$ & - & \\
\hline Median glb (g/L), (range) & $42.65(17.4-89.8)$ & $22.05(15.7-42.4)$ & 0.015 \\
\hline$\geq 35 \mathrm{~g} / \mathrm{L}$ & $6(50 \%)$ & $1(8.33 \%)$ & \\
\hline$<35 \mathrm{~g} / \mathrm{L}$ & $6(50 \%)$ & $11(91.67 \%)$ & \\
\hline $\begin{array}{l}\text { Median } \beta 2 \text {-microglobulin } \\
\text { (mg/L), (range) }\end{array}$ & $5.14(2.66-22.4)$ & $2.355(1.74-3.8)$ & 0.012 \\
\hline$\geq 5.5 \mathrm{mg} / \mathrm{L}$ & $6(50 \%)$ & - & \\
\hline$<5.5 \mathrm{mg} / \mathrm{L}$ & $6(50 \%)$ & $12(100 \%)$ & \\
\hline Median $\mathrm{Hb}(g / L)$, (range) & $107(67-140)$ & $124(100-137)$ & 0.059 \\
\hline$\geq 105 \mathrm{~g} / \mathrm{L}$ & $7(58.33 \%)$ & 11 (91.67\%) & \\
\hline$<105 \mathrm{~g} / \mathrm{L}$ & $5(41.67 \%)$ & $1(8.33 \%)$ & \\
\hline $\begin{array}{l}\text { Median Cre }(\mu \mathrm{mol} / \mathrm{L}) \text {, } \\
\text { (range) }\end{array}$ & $61.5(55-400)$ & $58.5(36-166)$ & 0.199 \\
\hline$\geq 104 \mu \mathrm{mol} / \mathrm{L}$ & $3(25 \%)$ & $1(8.33 \%)$ & \\
\hline$<104 \mu \mathrm{mol} / \mathrm{L}$ & $9(75 \%)$ & $11(91.67 \%)$ & \\
\hline $\begin{array}{l}\text { Median } \mathrm{Ca}^{++}(\mathrm{mmol} / \mathrm{L}) \\
\text { (range) }\end{array}$ & $2.305(1.95-4.19)$ & $2.145(1.87-2.28)$ & 0.087 \\
\hline$\geq 2.54 \mathrm{mmol} / \mathrm{L}$ & $3(25 \%)$ & - & \\
\hline$<2.54 \mathrm{mmol} / \mathrm{L}$ & $9(75 \%)$ & $12(100 \%)$ & \\
\hline LDH(U/L) (range) & $170(145-300)$ & $202(173-266)$ & 0.342 \\
\hline Normal & $10(83.33)$ & $9(75 \%)$ & \\
\hline High & $2(16.67 \%)$ & $3(25 \%)$ & \\
\hline \multicolumn{4}{|l|}{ M protein subtype, $n(\%)$} \\
\hline lgG kappa & $1(8.33)$ & & \\
\hline IgG lamda & $2(16.67 \%)$ & & \\
\hline IgA kappa & $3(25 \%)$ & & \\
\hline IgA lamda & $2(16.67 \%)$ & & \\
\hline Kappa light chain & $2(16.67 \%)$ & & \\
\hline Lamda light chain & $2(16.67 \%)$ & & \\
\hline $\begin{array}{l}\text { Median plasma cells in } \\
\text { bone marrow (\%), (range) }\end{array}$ & $20(0.5-58)$ & $2(0-11)$ & 0.002 \\
\hline
\end{tabular}

Characteristics of the patients included in the study. PCD: Bortezomib + Cyclophosphamide + Dexamethasone

rhCCL2. We also performed flow cytometry to evaluate whether CCL2 affects the response of MM cells to bortezomib. Based on the results, bortezomib induced similar levels of apoptosis in MM cells (Supplementary Fig. 1E), suggesting that rhCCL2 does not affect the drug response of MM cells. To determine why rhCCL2 had little effect on MM cells, we measured CCR2 expression in human MM cell lines by flow cytometry and found scarce expression of CCR2 (Supplementary Fig. 1F). This finding indicates that increased levels of CCL2 in MM BM may play an important role via other cell components.

rhCCL2-treated $M \varphi s$ are more effective at protecting $M M$ cells from melphalan- and bortezomib-induced apoptosis

Our previous studies showed that BM-infiltrated M $\phi s$ can induce drug resistance in $\mathrm{MM}^{7}$. To determine whether CCL2 promotes this protective effect of M $\phi$ s, we treated M $\phi$ s with rhCCL2 for $24 \mathrm{~h}$ to generate rhCCL2M $\phi$ s. As shown in Fig. 2a, coculture of MM cells with M $\phi s$ protected MM cells from bortezomib- and melphalan-induced apoptosis, and rhCCL2-M $\phi$ s were more effective than M $\phi$ s. Flow cytometry also revealed that when MM cells were cocultured with rhCCL2-M $\phi s$, bortezomib induced fewer MM cells to express active caspase-3 than when cocultured with $\mathrm{M} \phi \mathrm{s}$ (Fig. 2b). Additionally, Western blotting showed that bortezomib treatment resulted in cleaved caspase- 3 and cleaved PARP in MM cells. However, when ARP-1 cells were cocultured with $M \phi s$, bortezomib-induced PARP and caspase- 3 cleavage were highly repressed, and rhCCL2$\mathrm{M} \phi \mathrm{s}$ were more effective at this repression (Fig. 2c). Taken together, these results demonstrate that rhCCL2 promotes $\mathrm{M} \phi$ s to protect myeloma cells from melphalanand bortezomib-induced apoptosis by inhibiting caspase activation.

\section{rhCCL2 polarized $M \varphi s$ toward the M2-like phenotype in vitro}

According to previous studies, $\mathrm{M} \phi \mathrm{s}$ in different states of polarization exert different functions on MM cells' survival and tumor growth ${ }^{19}$. To test our hypothesis that the polarization of $M \phi s$ is associated with their capacity to protect MM cells, we generated IL4-M $\phi$ s and LPS + IFN $\gamma-\mathrm{M} \phi s$ and evaluated their protective capacity. IL4M $\phi$ s expressed higher levels of the M2 surface marker CD206 than M $\phi$ s and LPS + IFN $\gamma-M \phi s$, indicating that these cells were in different polarization states (Supplementary Fig. 2A). Compared with cells cocultured with $\mathrm{M} \phi \mathrm{s}$ or LPS + IFN $\gamma-\mathrm{M} \phi \mathrm{s}$, fewer MM cells cocultured with IL4-M $\phi$ s underwent apoptosis induced by bortezomib (Supplementary Fig. 2B). This result demonstrates that $M 2$-like $M \phi s$ are more effective at protecting $M M$ cells from bortezomib-induced apoptosis.

We next sought to determine whether rhCCL2 is able to polarize M $\phi$ s toward the M2-like phenotype. Flow cytometry analysis of $\mathrm{M} \phi \mathrm{s}$ surface markers showed that rhCCL2 upregulated expression of CD206 and CD163 (classic markers of M2-like M $\phi s$ ) and downregulated that of CD86 (classic surface marker of M1-like M $\phi s$ ) (Fig. 2d). Moreover, based on qRT-PCR, rhCCL2-M $\phi s$ 


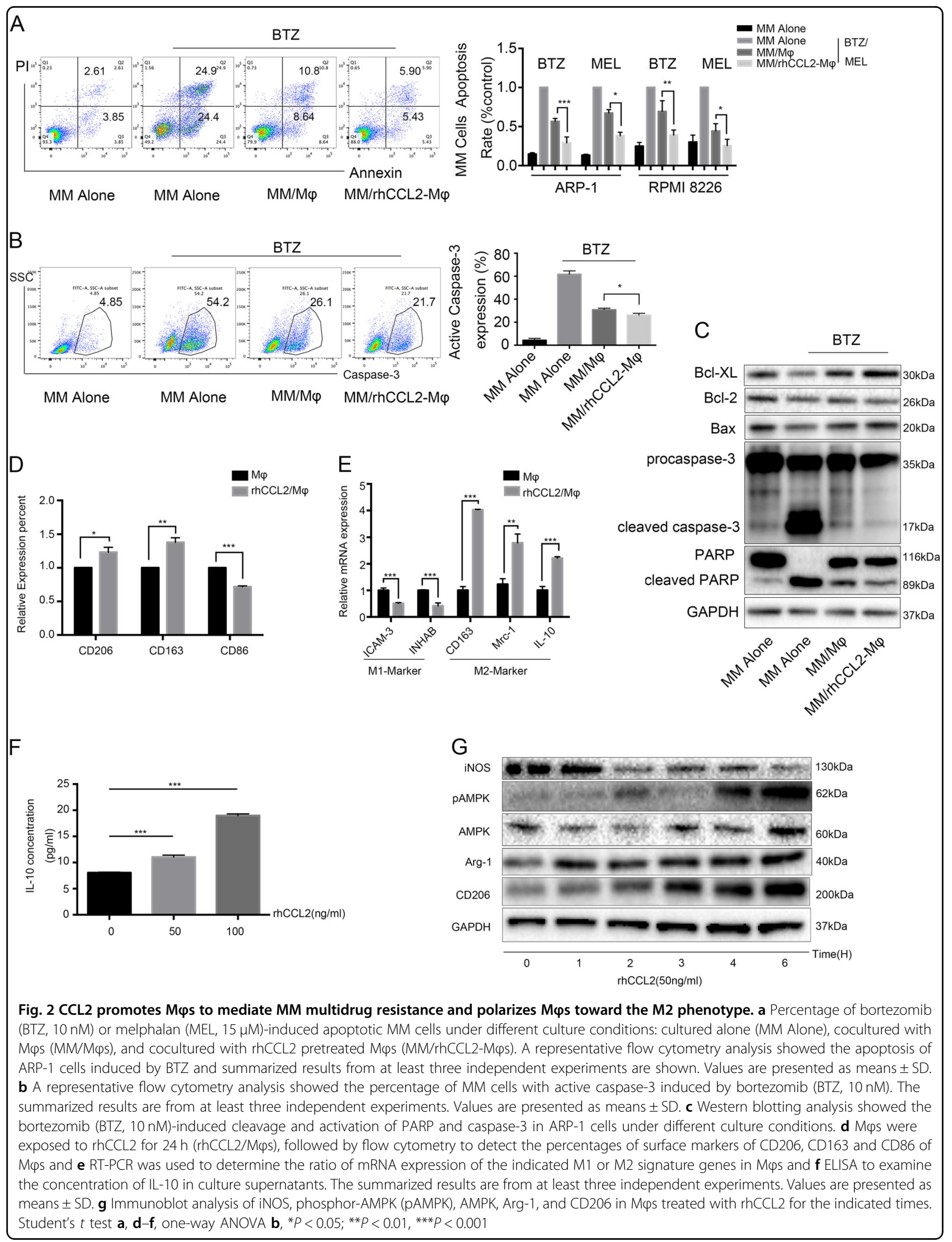


expressed lower mRNA levels of M1-like genes (ICAM-3 and INHAB) and higher levels of M2-like genes (CD163, Mrc-1, and IL-10) (Fig. 2e). ELISA was then conducted to detect secretion of IL-10, a classic anti-inflammation factor secreted by M2-like M $\phi$ s. As shown in Fig. 2f, rhCCL2-M $\phi$ s secreted more IL-10 than did untreated M $\phi$ s. In addition to changes in receptor surface expression and cytokine secretion, M $\phi$ s polarization was associated with a shift in energy metabolism, and adenosine $5^{\prime}$-monophosphate-activated protein kinase (AMPK) was central in this regulation ${ }^{19,21,22}$. These studies showed that M2-like M $\phi$ s were related to rapid AMPK phosphorylation and AMPK activation could drive IL-10 production in M $\phi s$. We next performed Western blotting and found that rhCCL2 time-dependently activated AMPK by increasing T172 phosphorylation levels. Expression of Arg-1 and CD206 was also upregulated along with the exposure of rhCCL2. All these results suggest that rhCCL2 effectively polarizes M $\phi$ s toward the M2-like phenotype, with a stronger ability to protect MM cells from bortezomib-and melphalan-induced apoptosis.

\section{A CCR2 inhibitor suppressed the protective effect of $M \varphi s$ in vivo}

Next, we performed in vivo experiments to determine the role of CCL2 in the MM microenvironment. Figure 3a shows the workflow of the experiment. CCX140-B is a specific CCR2 inhibitor, and as illustrated in Fig. 3b, c, there were no significant difference in the tumor volumes between the BTZ and CCX + BTZ groups, which indicated that blocking the CCL2-CCR2 axis in MM cells scarcely influenced the effect of bortezomib. However, in the presence of M $\phi s, C C X 140-B$ significantly hindered the growth of tumors, suggesting that blocking the CCL2-CCR2 axis in M $\phi$ s disrupted the protective effect of $M \phi s$ in vivo. Immunofluorescence results also revealed that the tumors in the $\mathrm{M} \phi+\mathrm{CCX}+\mathrm{BTZ}$ group had lower CD138 levels and higher active caspase-3 levels than in the $M \phi+B T Z$ group; thus, with CCX140-B treatment, more $\mathrm{MM}$ cells underwent apoptosis upon bortezomib treatment (Fig. 3d). We then explored the effect of CCR2 blockade on M $\phi$ s polarization by separating $\mathrm{CD} 14+$ cells from the tumor mass and using quantitative RT-PCR to detect expression of M $\phi s$ polarization-associated genes. The results showed that $\mathrm{M} \phi$ s from $\mathrm{M} \phi+\mathrm{CCX}+\mathrm{BTZ}$ group mice expressed significantly lower levels of IL-10, Arg-1, and Mrc-1, and higher levels of ICAM-3 and IL- $12 \alpha$ than did M $\phi$ s from $\mathrm{M} \phi+\mathrm{BTZ}$ group mice (Fig. 3e). Taken together, our results indicate that CCL2 is associated with the protective effect of $\mathrm{M} \phi \mathrm{s}$ and may alter the polarization status of these cells.

\section{CCL2 induced MCPIP1 expression in $M \varphi s$}

To explore the mechanism by which rhCCL2-M $\phi s$ are more effective at protecting MM cells, we cultured M $\phi s$ with rhCCL2 for $24 \mathrm{~h}$ and detected the global transcriptional profile of these cells by RNA sequencing (RNASeq). The change in some immune-related genes between M $\phi s$ and rhCCL2-M $\phi s$ is depicted in Fig. 4a. Among these genes, we were interested in the change of ZC3H12A, which is also called MCPIP1, has been uncovered to act as a negative regulator of inflammation $^{23}$. MCPIP1 was initially identified when gene expression changes in human PBMCs treated with CCL2 were analyzed by a genomic approach with gene arrays ${ }^{24}$, and this study named the most highly induced expressed sequence tag (EST) MCP-1-induced protein (MCPIP1). Considering the close connection between CCL2 and MCPIP1, we attempted to determine whether MCPIP1 is involved in the enhanced protective effect of rhCCL2$\mathrm{M} \phi \mathrm{s}$ or whether it promotes M2 polarization.

As shown in Fig. 4b-d, rhCCL2 induced expression of MCPIP1 in M $\phi s$. We also found that the culture medium of MM cells (RPMI 8226) significantly upregulated MCPIP1 expression in M $\phi$ s. In the presence of $\alpha C C L 2$, a neutralizing antibody, MCPIP1 expression was downregulated, indicating that increased CCL2 expression induced by MM cells also effectively triggered MCPIP1 expression in M $\phi$ s (Fig. 4e, f).

\section{The enhanced protective effect of rhCCL2-M $\varphi$ s relied on induced expression of MCPIP1}

To determine whether upregulated MCPIP1 expression promotes the observed protective effect, $M \phi s$ were transfected with MCPIP1-specific siRNA or a nonspecific scrambled control. As shown in Fig. 5a, when MM cells were cocultured with siMCPIP1-M $\phi$ s, the apoptosis rates induced by bortezomib or melphalan were much higher than those of cells cocultured with siNC-M $\phi$ s. This finding indicated that expression of MCPIP1 in M $\phi s$ affected their capacity to protect MM cells from melphalan- and bortezomib-induced apoptosis. Next, we conducted the same experiment using primary MM cells from a patient and found that siMCPIP1-M $\phi$ s possessed a poor capacity to protect primary MM cells upon bortezomib treatment (Supplementary Fig. 3A). In addition, we performed flow cytometry to assess the percentage of MM cells with activated caspase- 3 induced by bortezomib (Fig. $5 \mathrm{~b})$, and the results indicated that knocking down MCPIP1 hindered the M $\phi s-$ mediated protection of MM cells from bortezomib-induced apoptosis. Moreover, Western blotting revealed that compared with siNC-M $\phi$, siMCPIP1-M $\phi$ s were inferior in suppressing bortezomibinduced activation and cleavage of caspase- 3 and PARP in MM cells (Fig. 5c). 


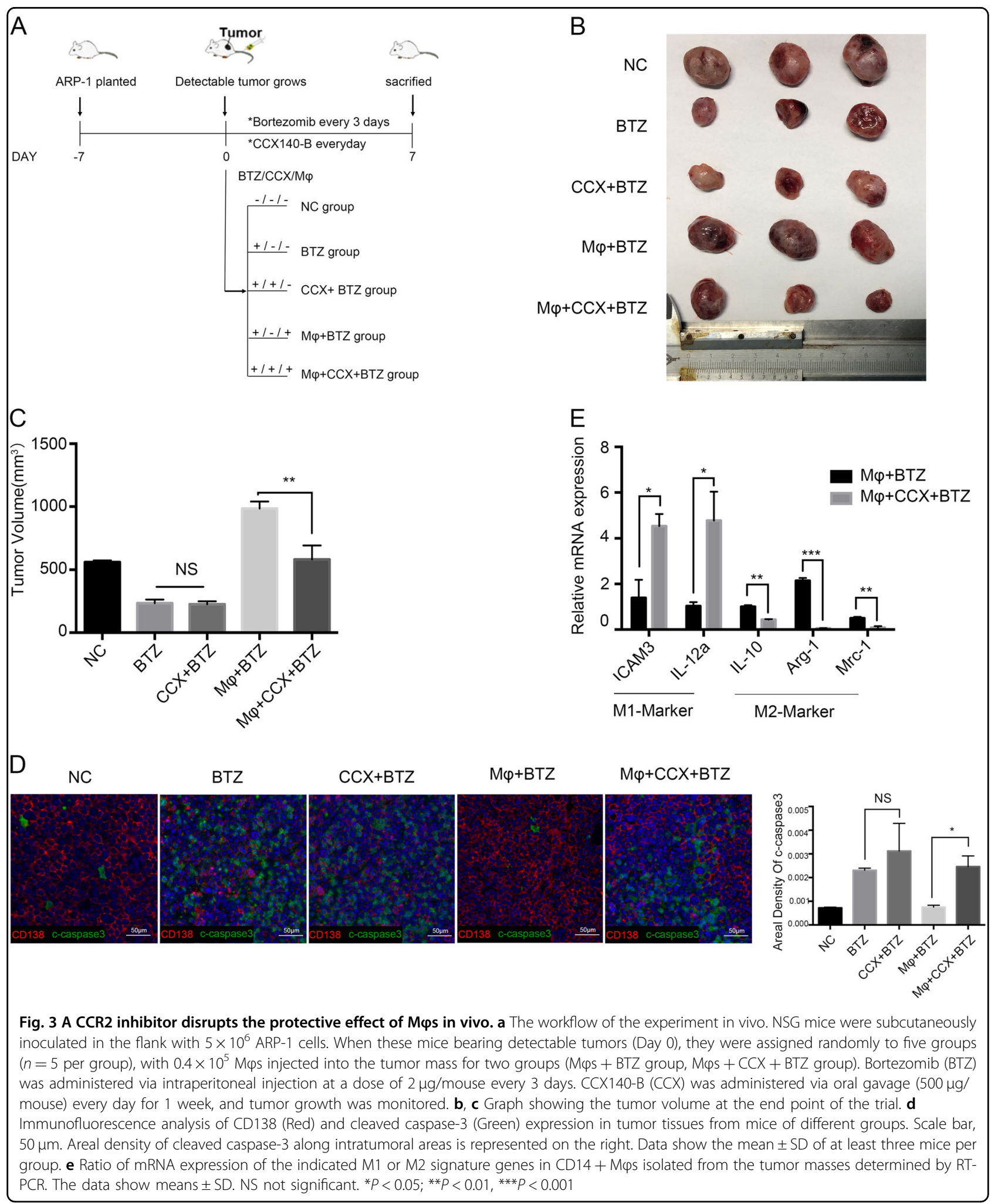

To rule out the possibility that transfection with the MCPIP1-specific siRNA affected M $\phi$ s survival, we conducted flow cytometry and Western blotting to assess apoptosis in siMCPIP1-M $\phi$ s and siNC-M $\phi$ s. As shown in
Supplementary Fig. 3B, C, there were no differences in apoptosis between these cells, indicating that transfection with the MCPIP1-specific siRNA barely affected M $\phi s$ survival. 


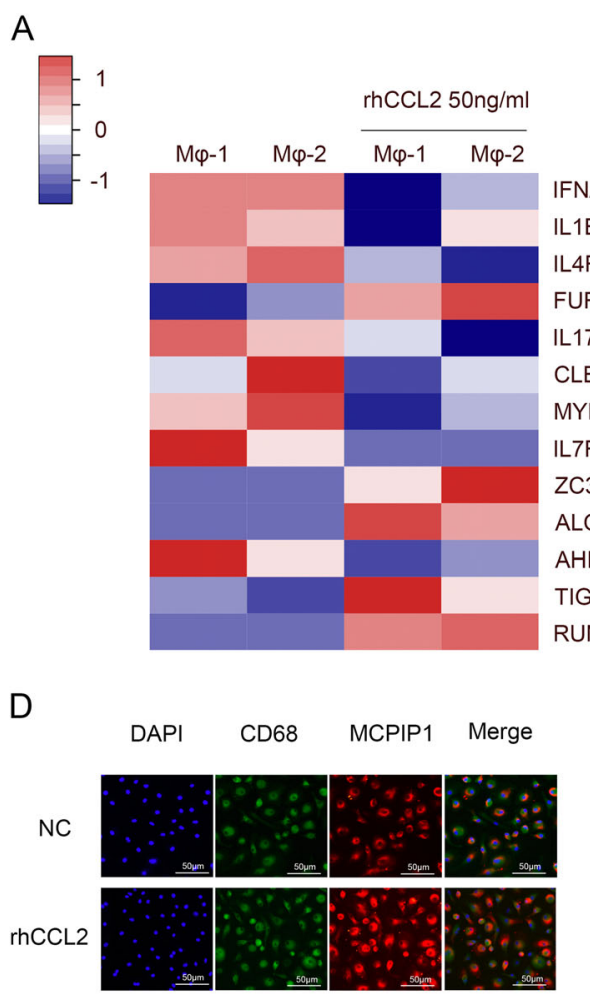

\section{B}

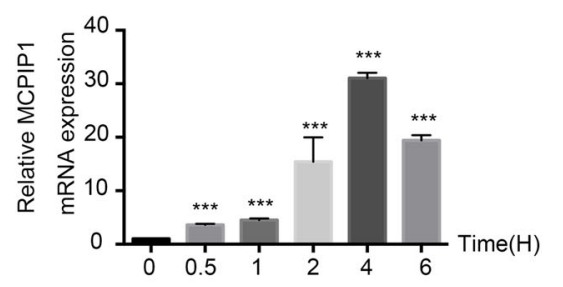

CLEC5A

MYD88

IL7R

ZC3H12A

ALOX15

AHNAK

TIGIT

RUNX2
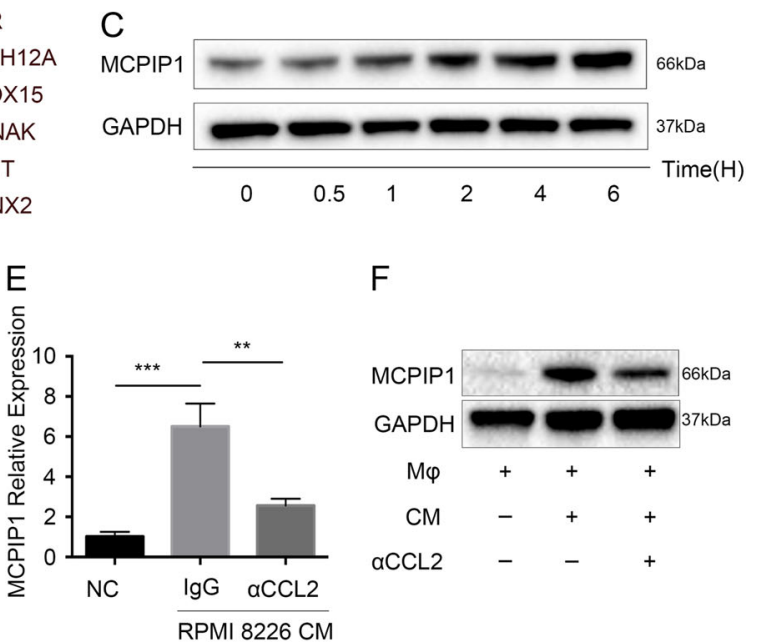

F

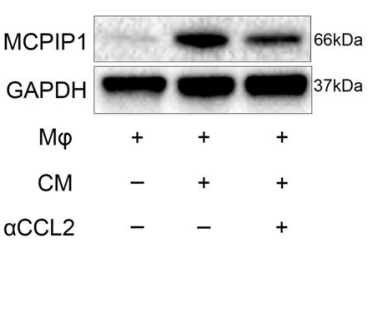

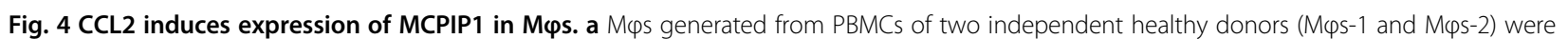
treated with rhCCL2 $(50 \mathrm{ng} / \mathrm{ml})$ for $24 \mathrm{~h}$. RNA was extracted for RNA sequencing. Heat maps illustrate the $\log _{2}$-fold change of some immune-related genes. b M $\varphi s$ were exposed to rhCCL2 $(50 \mathrm{ng} / \mathrm{mL})$ for the indicated times, followed by RT-PCR and Western blotting $\mathbf{c}$ to determine expression of MCPIP1. d M $\varphi s$ were treated with rhCCL2 $(50 \mathrm{ng} / \mathrm{mL})$ for $24 \mathrm{~h}$. Immunofluorescence was performed to detect expression of MCPIP1 in M $\varphi s$. Scale bar, $50 \mu \mathrm{m}$. e, f M $\mathrm{M}$ s were treated with RPMI-8226 CM for $24 \mathrm{~h}$ with or without aCCL2. qRT-PCR e and Western blotting $\mathbf{f}$ were performed to detect MCPIP1 expression. Data are presented as means \pm SD of at least three independent experiments. ${ }^{*} P<0.05 ;{ }^{* *} P<0.01,{ }^{* * *} P<0.001$

\section{MCPIP1 promoted the protective effect of M $\varphi$ s via dual catalytic activities}

MCPIP1 is known to have deubiquitinase and RNase activities, including anti-Dicer activity ${ }^{25-27}$. To determine whether these activities of MCPIP1 are involved in the enhanced protective effect of $M \phi s$, we used lentiviruses to generate deletion mutants of MCPIP1. It has been shown that the D141N mutant of MCPIP1 inactivates both its RNase and DUB activities ${ }^{28}$. As shown in Fig. 5d, M $\phi$ s transfected with wild-type MCPIP1 exhibited better protective effects than did $M \phi s$ transfected with the lentivirus vector. Interestingly, when ARP-1 cells were cocultured with D141N-mutant MCPIP1 M $\phi$ s, more MM cells underwent apoptosis than when these cells were cocultured with wild-type MCPIP1 M $\phi$ s, and the apoptosis rates were as high as those when the cells were cocultured with $M \phi s$ transfected with the lentivirus vector. These results indicate that MCPIP1 overexpression promotes the protective effect of $M \phi s$ via its dual catalytic activities.
The protective effect of M $\varphi$ s relied on MCPIP1 expression in vivo

We next used an MM cell xenograft model to determine whether MCPIP1 plays an important role in promoting $\mathrm{M} \phi \mathrm{s}$ to protect MM cells in vivo. Figure 6a shows the workflow of the experiment. ARP-1 cells were first injected subcutaneously into NSG mice, and when the average tumor volume reached approximately $5 \mathrm{~mm}^{3}$, we injected either siNC-M $\phi$ s or siMCPIP1-M $\phi$ s into the tumor mass. In addition, the mice were treated with bortezomib every three days. As shown in Fig. 6b, c, tumor size measurements revealed that the siNC-M $\phi$ group developed larger tumors than the siMCPIP1-M $\phi$ group, suggesting that MCPIP1 has an important role in the protective effect of M $\phi$ s. Furthermore, tissue analysis showed that MM + siMCPIP1-M $\phi$ tumors displayed low CD138 levels and high active caspase-3 levels. Consequently, mice in this group had a lighter tumor burden, and more myeloma cells underwent apoptosis upon 


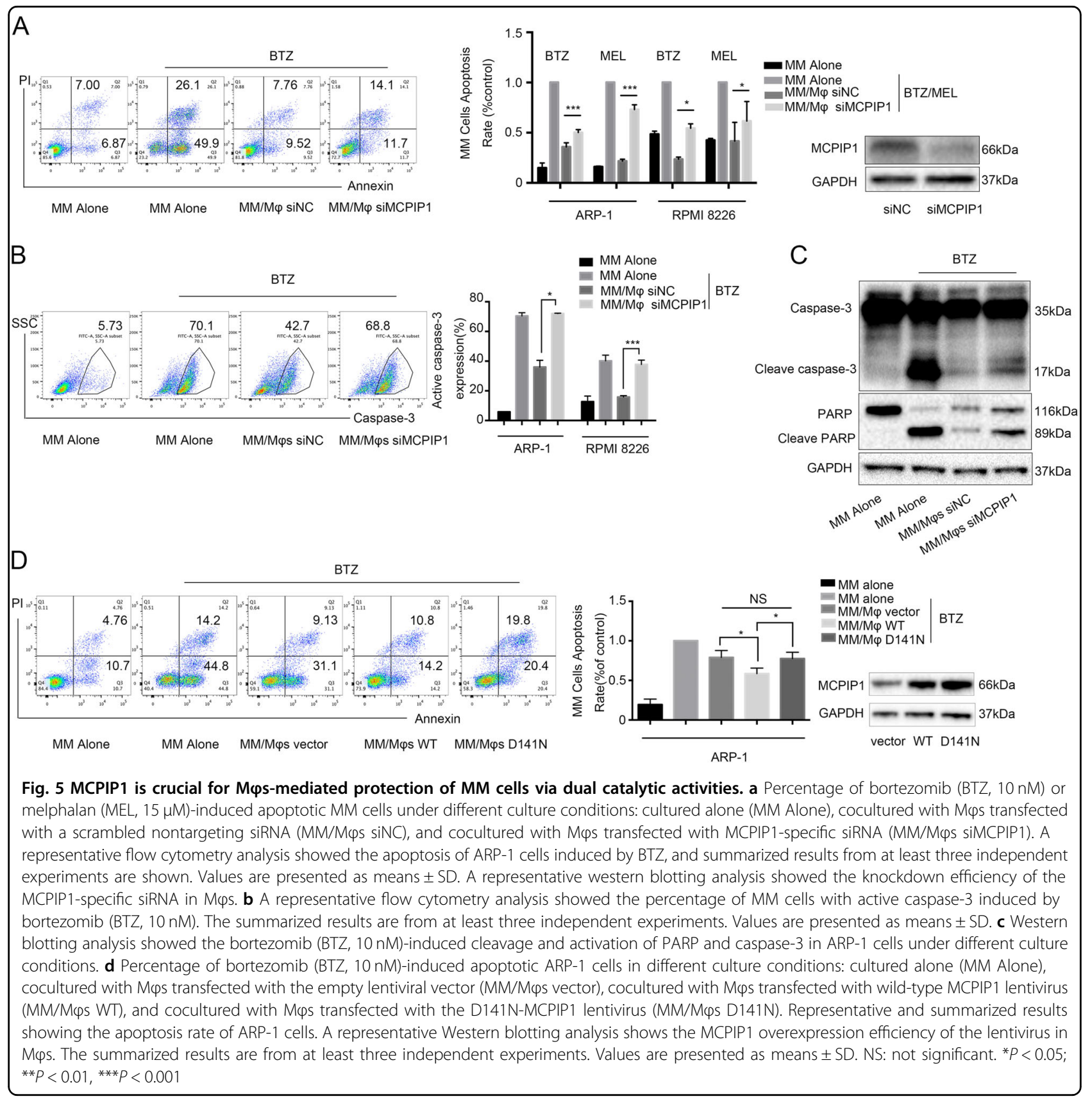

bortezomib treatment (Fig. 6d). These data demonstrate that the protective effect of M $\phi s$ in vivo relies on MCPIP1.

\section{MCPIP1 was crucial for M $\varphi$ s polarization}

To compare the polarization state of siNC-M $\phi$ s and siMCPIP1-M $\phi$ s in vivo, we isolated leukocytes from the NSG mouse tumor masses and then conducted quantitative RT-PCR to determine the polarization state of M $\phi$ s. As shown in Fig. 6e, siMCPIP1-M $\phi$ s expressed greater mRNA levels of M1 M $\phi$ s-associated IL-12 $\alpha$ and TNF- $\alpha$ and lower levels of M2 M $\phi s$-associated Mrc-1, IRF4, and IL-10. We also performed flow cytometry to detect
CD206 expression on CD14+ cells and found that siMCPIP1-M $\phi$ s expressed less CD206 than did siNC-M $\phi s$ (Fig. 6f). Taken together, the results suggest that MCPIP1 is crucial for $M \phi s$ polarization in vivo.

We also explored the role of MCPIP1 in M $\phi s$ polarization in vitro. As shown in Fig. 6g, siMCPIP1-M $\phi$ s expressed much less CD206 than did siNC-M $\phi$ s. In addition, expression of CD206 was upregulated when siNC-M $\phi$ s were cocultured with ARP-1 cells, though expression barely changed when siMCPIP1-M $\phi$ s were cocultured with ARP-1 cells. These data suggest that M $\phi s$ with MCPIP1 knockdown not only display a more M1- 


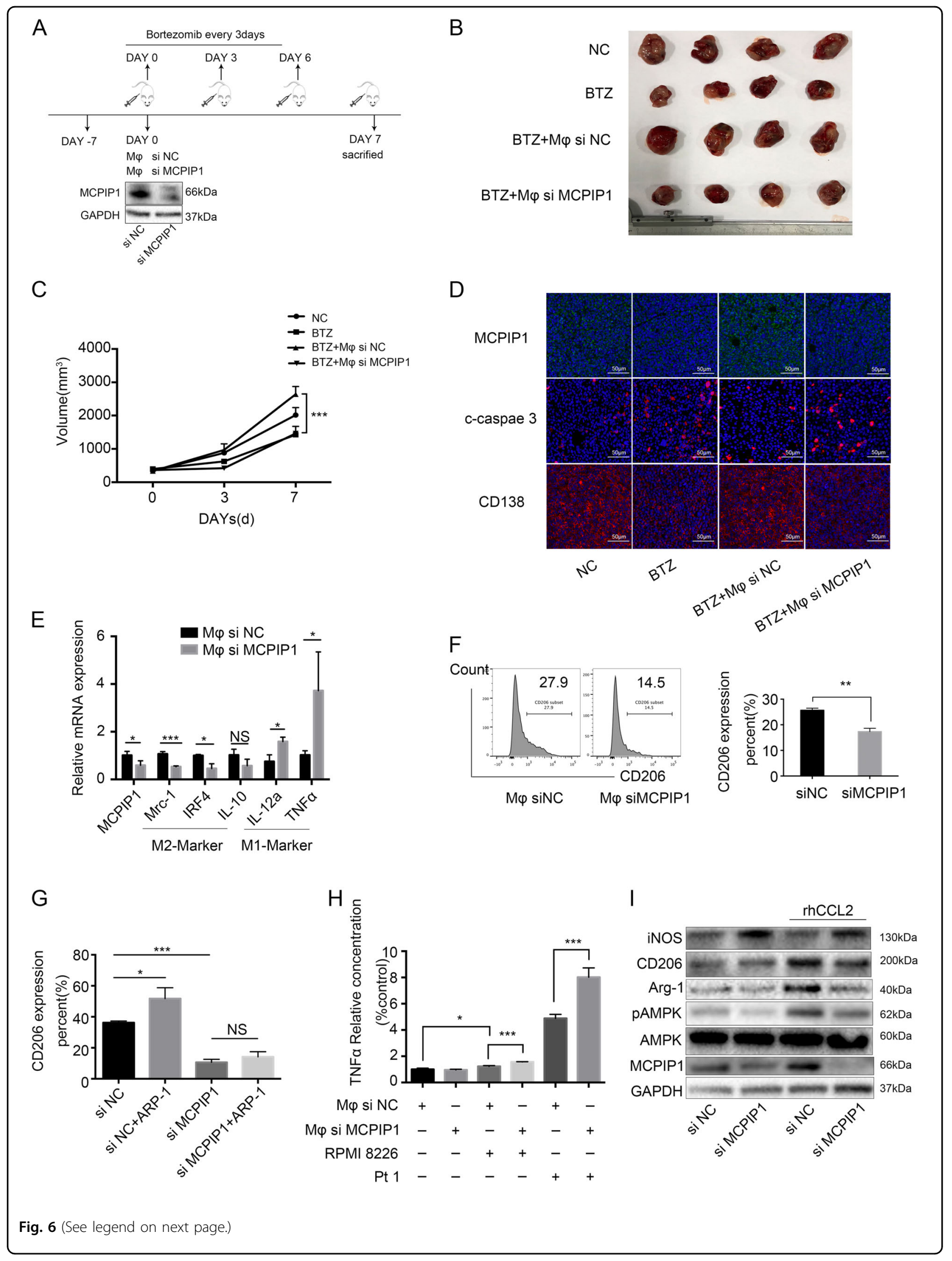


(see figure on previous page)

Fig. 6 The protective effect of $M \varphi s$ relies on MCPIP1 in vivo, and MCPIP1 is crucial for M $\varphi$ s polarization. a The workflow of the experiment in vivo. NSG mice were subcutaneously inoculated in the flank with $5 \times 10^{6}$ ARP-1 cells. When tumors were detectable (Day 0 ), the mice were assigned randomly to 4 groups ( $n=6$ per group), with M $\varphi s$ that were differentially transfected $\left(0.4 \times 10^{5}\right)$ injected into the tumor mass for two

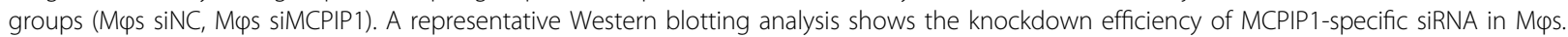
Bortezomib (BTZ) was administered via intraperitoneal injection at a dose of $2 \mu \mathrm{g} / \mathrm{mouse}$ every 3 days, and tumor growth was monitored. $\mathbf{b} \mathrm{A}$ representative image of tumor volumes at the end point of the trial c Mean tumor volumes over the time course. $\mathbf{d}$ Immunofluorescence analysis of MCPIP1, CD138, and cleaved caspase-3 expression in tumor tissues from mice of different groups. Scale bar, $50 \mu \mathrm{m}$. e Ratio of mRNA expression of the

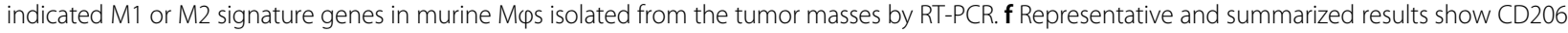
expression measured by flow cytometry in murine M $\varphi$ s isolated from the tumor mass. Values are presented as means \pm SD. $\mathbf{g}$ Differently transfected M $\varphi s$ (siNC, siMCPIP1) were cocultured with or without ARP-1 cells for $24 \mathrm{~h}$, followed by flow cytometry to detect CD206 expression. The summarized results are from at least three independent experiments. Values are presented as means \pm SD. $\mathbf{h}$ Differently transfected M $\varphi s$ (siNC, siMCPIP1) were cocultured with or without RMPI 8226 cells and primary MM cells from a patient (Pt.1) for $24 \mathrm{~h}$. The fresh medium was changed, and M $\varphi s$ were cultured for another $24 \mathrm{~h}$, followed by ELISA analysis to detect TNF-a expression. The summarized results are from at least three independent experiments. Values are presented as means \pm SD. i Differently transfected M $\varphi s$ (siNC, siMCPIP1) were exposed to $\mathrm{rhCCL} 2(50 \mathrm{ng} / \mathrm{mL}$ ) for $24 \mathrm{~h}$, and immunoblot analysis was conducted to determine expression of iNOS, CD206, Arg-1, pAMPK, AMPK, and MCPIP1. The data show means \pm SD. NS not significant. ${ }^{*} P<0.05 ;{ }^{*} P<0.01,{ }^{* * *} P<0.001$

like phenotype but that they are also more difficult to be polarized toward the M2-like phenotype by MM cells.

As previous studies have shown that TNF- $\alpha$, a cytotoxic factor, is involved in the tumoricidal effect of $\mathrm{M} \varphi \mathrm{s}^{19}$, we then performed ELISA to detect secretion of TNF- $\alpha$ by $\mathrm{M} \phi \mathrm{s}$. The results indicated that although siNC-M $\phi$ s and siMCPIP1-M $\phi$ s secreted little TNF- $\alpha$, this secretion was upregulated when M $\phi$ s were cocultured with RMPI.8226 cells and primary MM cells. In addition, siMCPIP1-M $\phi$ secretion was significantly higher than that of siNC-M $\phi s$ when cocultured with MM cells (Fig. 6h). According to Western blot analysis, knocking down MCPIP1 downregulated expression of Arg-1, CD206, and PAMPK and upregulated that of iNOS. In addition, rhCCL2 induced expression of Arg-1, CD206, and pAMPK and suppressed that of iNOS in siNC-M $\phi$ s, whereas rhCCL2 had little effect on siMCPIP1-M $\phi s$ (Fig. 6i).

All of these in vitro experiments indicate that MCPIP1 is crucial for $M \phi s$ polarization.

\section{CCL2-induced MCPIP1 expression was dependent on the JAK2-STAT3 pathway}

Finally, we used Proteome Profiler Human Phosphokinase Antibody Array to determine changes in protein phosphorylation in M $\phi$ s caused by rhCCL2. The results showed that CCL2 significantly activated STAT3 by increasing S727 phosphorylation levels in M $\phi$ s (Fig. 7a). We next performed Western blotting to confirm this activation, and as shown in Fig. 7b, rhCCL2 resulted in STAT3 phosphorylation at $4 \mathrm{~h}$ after exposure, and rhCCL2 also phosphorylated S727-STAT3 in a concentration-dependent manner (Fig. 7c). Furthermore, MM cell conditioned medium activated phosphorylation of STAT3 (S727), and the CCR2 inhibitor CCX140-B inhibited this activation (Fig. 7d). To verify the role of STAT3 in CCL2-induced MCPIP1 expression, we blocked the STAT3 pathway with the STAT3 inhibitor Stattic. As depicted in Fig. 7e, Stattic indeed inhibited STAT3 phosphorylation in M $\phi$ s induced by rhCCL2 and ARP-1 cells and significantly downregulated CCL2-induced MCPIP1 protein expression.

Taken together, the results indicate that CCL2 induces MCPIP1 expression via the JAK2-STAT3 signaling pathway in $M \phi s$.

\section{Discussion}

The mechanism of MM chemoresistance is associated with both intrinsic changes in MM cells and the protective efficiency of $\mathrm{BMSCs}^{3}$. M $\phi s$, a prominent component in the BM microenvironment of MM, play an important role in protecting MM cells from drug-induced apoptosis.

We previously reported that CCL2 expression was increased in the $\mathrm{BM}$ of $\mathrm{MM}$ patients and promotes $\mathrm{M} \phi \mathrm{s}$ infiltration into the $\mathrm{BM}^{13}$. In this study, we demonstrated that $M \phi s$ abundantly express CCL2; we also found that coculture with $\mathrm{MM}$ cells further upregulates $\mathrm{M} \phi \mathrm{s}^{\prime}$ expression of CCL2. To examine why M $\phi$ s upregulated expression of CCL2 when cocultured with MM cells, which is similar to the MM BM microenvironment, we performed mechanism studies to further elucidate the role of increased CCL2 expression in the BM microenvironment.

Interestingly, we found that when newly diagnosed patients received four courses of PCD combined therapy, CCL2 expression in their BM was greatly decreased. According to their clinicopathologic characteristics, almost all of these patients have been in remission due to therapy. Thus, CCL2 expression is tightly related to MM patient treatment status.

CCL2 has long been recognized as a regulator of TAMs in different cancers ${ }^{29-31}$. Some tumor cells also express CCL2, and high-CCL2 expression is associated with tumor cell therapy resistance ${ }^{32,33}$. However, in our study, MM cells barely expressed CCL2, and CCL2 treatment had little 

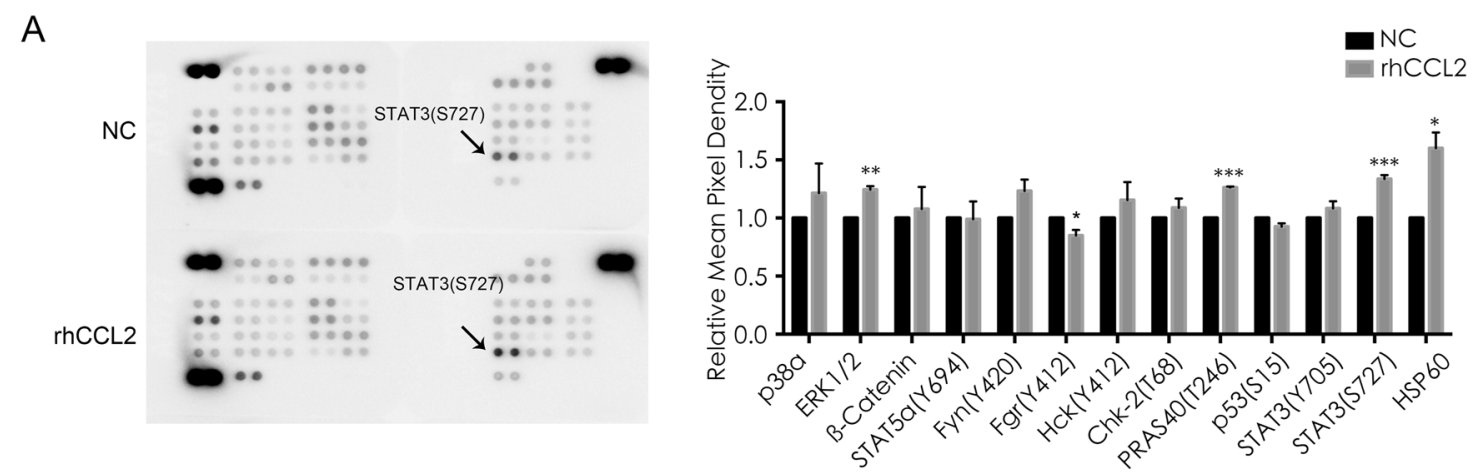

B
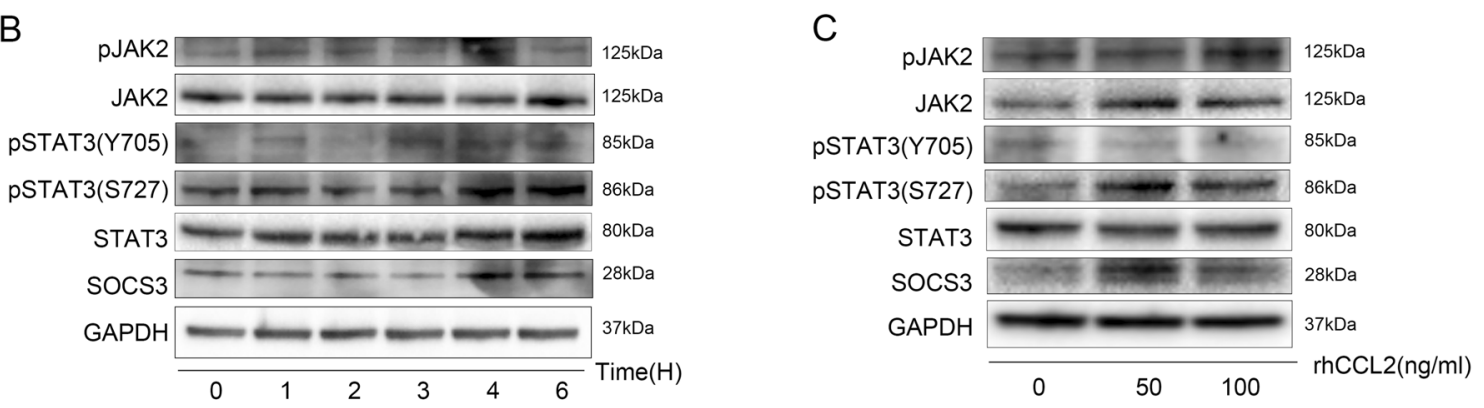

D

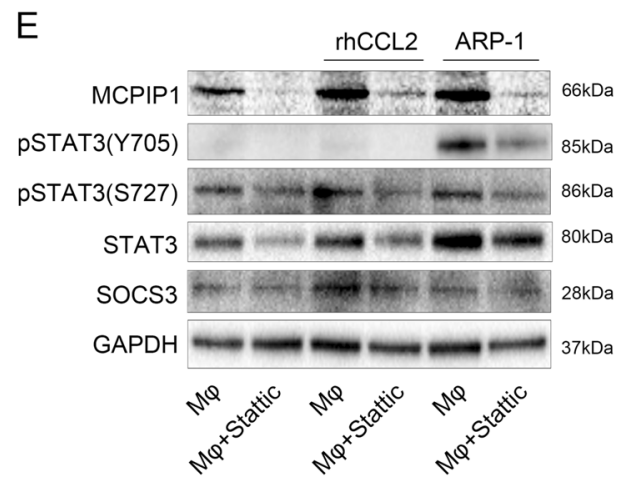

Fig. 7 CCL2 induces MCPIP1 expression through the JAK2-STAT3 pathway (a) M 9 s were treated with or without rhCCL2 (50 ng/mL) for $24 \mathrm{~h}$ and then harvested for protein level detection using Proteome Profiler Human Phosphorylation Kinase Antibody Array. Each target was assayed in duplicate. Representative images and quantitative diagrams of the relative content of each protein are expressed as the fold change of the blot intensity normalized to that of the NC group. b, c Western blotting results for expression of phosphorylated STAT3, JAK2, and SOCS3 in M $\varphi$ S stimulated with rhCCL2 for the indicated time and concentration. $\mathbf{d}$ M $\varphi$ s were exposed to RPMI-8226 conditioned medium (CM) for the indicated time with or without CCX140-B (CCX). Western blotting was applied to detect STAT3 phosphorylation. e M $\varphi$ s were treated with or without the STAT3 inhibitor Stattic $(20 \mu \mathrm{M})$ for $2 \mathrm{~h}$ and then exposed to rhCCL2 $(50 \mathrm{ng} / \mathrm{mL})$ or cocultured with ARP-1 cells for $24 \mathrm{~h}$. Western blotting was conducted to determine MCPIP1 expression and phosphorylation of STAT3, STAT3, and SOCS3 in M $\varphi$ S. Similar results were observed in at least three independent experiments

effect on MM cells' survival or proliferation. We showed that CCL2 is important for the protective effect of M $\phi$ s on MM cells. In addition, we found that CCL2 could skew the M $\phi$ s phenotype toward the M2-like phenotype, which is consistent with the results of some studies in solid tumors $^{34}$. We also found that $M \phi s$ with different polarization statuses exert different protective effects on MM cells, which indicates that CCL2 promotes the protective effect of $\mathrm{M} \phi s$ by polarizing them toward the M2-like phenotype.
Based on mechanistic studies, CCL2 induces expression of MCPIP1 in M $\phi$ s. MCPIP1 participates in M $\phi$ s polarization and promote the protective capacity of these cells via its dual catalytic activities. We also found that CCL2 induces expression of MCPIP1 via the JAK2-STAT3 signaling pathway. Overall, this study provides the first direct evidence that CCL2 induces $M \phi s$ polarization toward the M2-like phenotype and promotes their protective effects on MM cells via MCPIP1. 


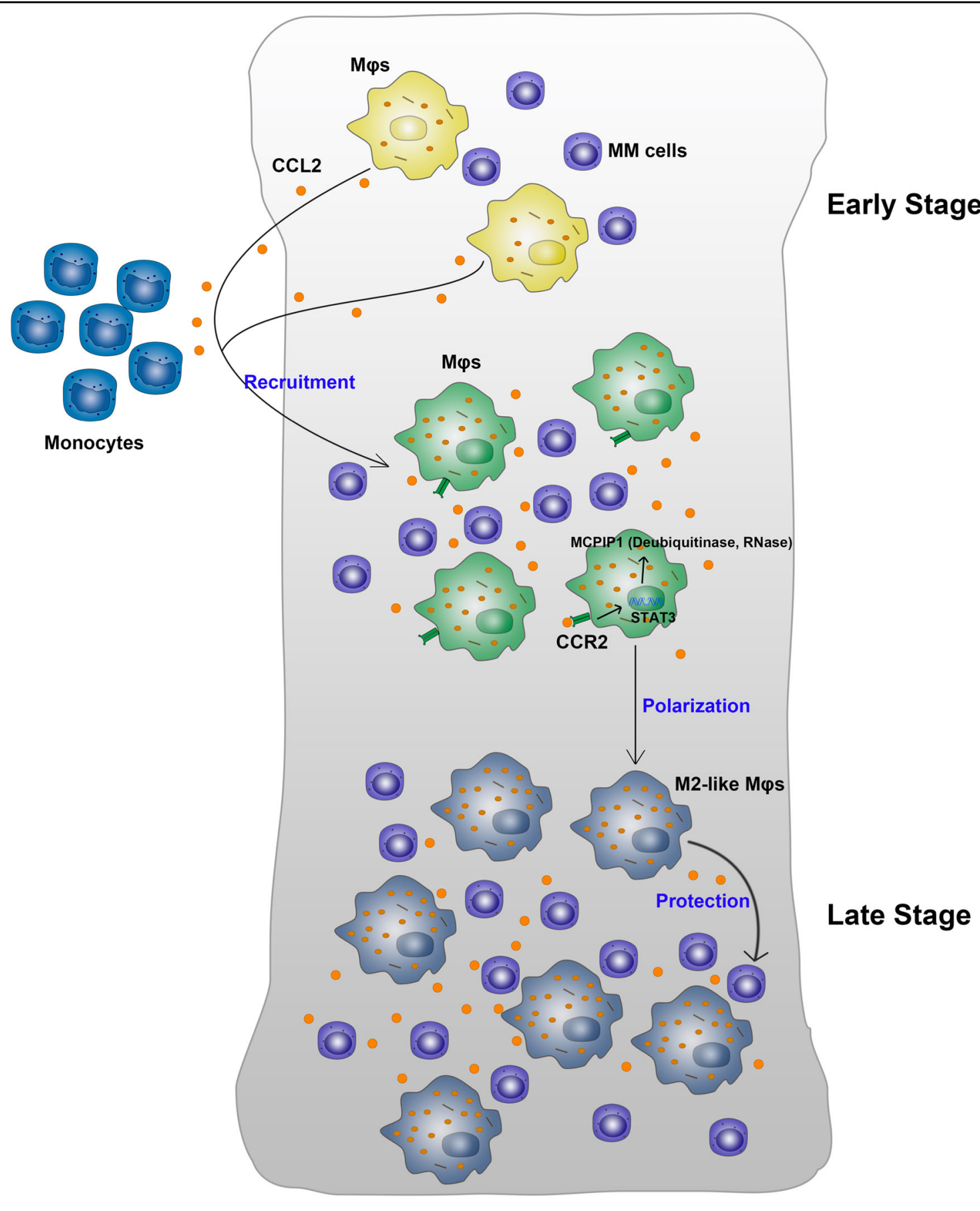

\section{Bone Marrow}

Fig. 8 A summarizing figure of main findings presented in the study. CCL2 strongly recruits monocytes to the BM microenvironment. Then

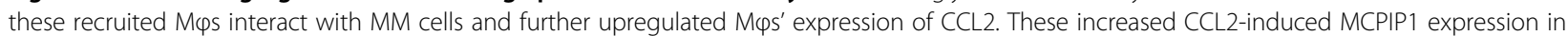

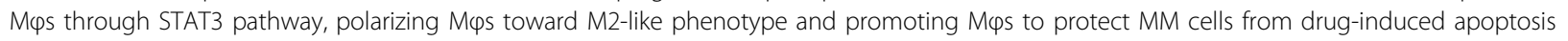

Interaction of CCL2 with its receptor CCR2 causes signal transduction events that induce the zinc-finger protein MCPIP1, and many studies have revealed that
MCPIP1 is a critical immunoregulatory agent. MCPIP1 is essential for preventing aberrant T-cell activation ${ }^{23}$, and it was also reported that MCPIP1 participates in IL4- 
induced $M \phi$ s polarization $^{35}$. Our data show that MCPIP1 indeed enhances the protective effect of $M \phi s$ and is crucial for $\mathrm{M} \phi$ s polarization. Although we found that the protective effect of $\mathrm{M} \phi \mathrm{s}$ is associated with the dual catalytic activities of MCPIP1, the most important functional domain and specific substrate that mediates the $M \phi s^{\prime}$ effect is not fully defined and requires further study.

Monoclonal antibody-based therapeutics have great promise for many tumors. For example, carlumab, a human IgG1k anti-CCL2 mAb, has shown antitumor activity in preclinical trials; however, another study showed that carlumab was ineffective at yielding longlasting decreases in serum CCL2 concentrations and thus had minimal clinical effects ${ }^{36}$. Because of the relationship between CCL2 expression and MM patient treatment status, we speculated that CCL2 might act as an effective prognostic factor for $\mathrm{MM}$ patients. In addition, new therapeutic strategies targeting MCPIP1 are likely to be promising.

In summary, CCL2 strongly recruits $M \phi s$ to the BM microenvironment. These recruited $M \phi s$ then interact with MM cells and further upregulate their expression of CCL2. The increased levels of CCL2 in the microenvironment polarize $\mathrm{M} \phi s$ toward the M2-like phenotype and promote $\mathrm{M} \phi \mathrm{s}$ to protect MM cells from chemotherapy drug-induced apoptosis (Fig. 8). Mechanistically, CCL2 induces expression of MCPIP1, a critical negative regulator of inflammation that mediates $M \phi s$ polarization and protection via its dual catalytic activities. This study provides new insight into the mechanism of drug resistance in MM.

\section{Acknowledgements}

This work was supported by National Natural Science Foundation of China (Project nos. 81700201 and 81770217), Natural Science Foundation of Zhejiang Province (Project nos. LY17H080001 and LY18H310014).

\section{Authors' contributions}

Z.C. and Y.L. initiated the study. Z.C., Y.L. and R.X. designed the experiments. R.X. and Y.L. performed the majority of the experiments. R.X. wrote the paper. H.Y., E.Z., X.H., Q.C. and J.C. performed the research and analyzed the data. J.Q. and Y.L. collected primary samples for the study. J.H., Q.Y. and Z.C. supervised the experiments.

\section{Conflict of interest}

The authors declare that they have no conflict of interest.

\section{Publisher's note \\ Springer Nature remains neutral with regard to jurisdictional claims in published maps and institutional affiliations.}

Supplementary Information accompanies this paper at (https://doi.org/ 10.1038/s41419-019-2012-4)

Received: 21 June 2019 Revised: 27 August 2019 Accepted: 19 September 2019

Published online: 14 October 2019

\section{References}

1. Kumar, S. K. et al. Multiple myeloma. Nat. Rev. Dis. Prim. 3, 17046 (2017).

2. Anderson, K. C. Progress and paradigms in multiple myeloma. Clin. Cancer Res. 22, 5419-5427 (2016)

3. Lohr, J. G. et al. Widespread genetic heterogeneity in multiple myeloma: implications for targeted therapy. Cancer Cell. 25, 91-101 (2014).

4. Hideshima, T., Mitsiades, C., Tonon, G., Richardson, P. G. \& Anderson, K. C. Understanding multiple myeloma pathogenesis in the bone marrow to identify new therapeutic targets. Nat. Rev. Cancer 7, 585-598 (2007).

5. Podar, K., Chauhan, D. \& Anderson, K. C. Bone marrow microenvironment and the identification of new targets for myeloma therapy. Leukemia $\mathbf{2 3}, 10-24$ (2009).

6. Glavey, S. V. et al. Proteomic characterization of human multiple myeloma bone marrow extracellular matrix. Leukemia 31, 2426-2434 (2017).

7. Zheng, Y. et al. Macrophages are an abundant component of myeloma microenvironment and protect myeloma cells from chemotherapy druginduced apoptosis. Leukemia 114, 3625-3628 (2009).

8. Zheng, Y. et al. PSGL-1/selectin and ICAM-1/CD18 interactions are involved in macrophage-induced drug resistance in myeloma. Leukemia 27, 702-710 (2013).

9. Murray, P. J. Macrophage polarization. Annu Rev. Physiol. 79, 541-566 (2017).

10. Sica, A. \& Mantovani, A. Macrophage plasticity and polarization: in vivo veritas. J. Clin. Invest. 122, 787-795 (2012).

11. Singhal, S. et al. Human tumor-associated monocytes/macrophages and their regulation of T cell responses in early-stage lung cancer. Sci. Transl. Med. 11, 479 (2019).

12. Martínez, V. G. et al. BMP4 induces M2 macrophage polarization and favors tumor progression in bladder cancer. Clin. Cancer Res. 23, 7388-7399 (2017).

13. Li, $Y$. et al. Chemokines CCL2, 3, 14 stimulate macrophage bone marrow homing, proliferation, and polarization in multiple myeloma. Oncotarget 6 24218-24229 (2015)

14. Kolattukudy, P. E. \& Niu, J. Inflammation, endoplasmic reticulum stress, autophagy, and the monocyte chemoattractant protein-1/CCR2 pathway. Circ. Res. 110, 174-189 (2012).

15. Castela, M. et al. $\mathrm{Cl} 2 / \mathrm{Ccr} 2$ signalling recruits a distinct fetal microchimeric population that rescues delayed maternal wound healing. Nat. Commun. 8, 15463 (2017).

16. Raghu, $H$. et al. CCL2/CCR2, but not CCL5/CCR5, mediates monocyte recruitment, inflammation and cartilage destruction in osteoarthritis. Ann. Rheum. Dis. 76, 914-922 (2017).

17. Bartneck, M. et al. The CCR2 macrophage subset promotes pathogenic angiogenesis for tumor vascularization in fibrotic livers. Cell Mol. Gastroenterol. Hepatol. 7, 371-390 (2019).

18. Ohba, T. et al. Bisphosphonates inhibit osteosarcoma-mediated osteolysis via attenuation of tumor expression of MCP-1 and RANKL. J. Bone Min. Res. 29, 1431-1445 (2014).

19. Gutiérrez-González, A. et al. Evaluation of the potential therapeutic benefits of macrophage reprogramming in multiple myeloma. Blood 128, 2241-2252 (2016).

20. Matsui, $H$. et al. M1 macrophages are predominantly recruited to the major pelvic ganglion of the rat following cavernous nerve injury. J. Sex. Med. 14, 187-195 (2017).

21. Kelly, B. \& O'Neill, L. A. Metabolic reprogramming in macrophages and dendritic cells in innate immunity. Cell Res. 25, 771-784 (2015).

22. Sag, D., Carling, D., Stout, R. D. \& Suttles, J. AMP-activated protein kinase promotes macrophage polarization to an anti-inflammatory functional phenotype. J. Immunol. 181, 8633-9641 (2008).

23. Xu, J., Fu, S., Peng, W. \& Rao, Z. MCP-1-induced protein-1, an immune regulator. Protein Cell. 3, 903-910 (2012).

24. Zhou, L. et al. Monocyte chemoattractant protein-1 induces a novel transcription factor that causes cardiac myocyte apoptosis and ventricular dysfunction. Circ. Res. 98, 1177-1185 (2006).

25. Fu, M. \& Blackshear, P. J. RNA-binding proteins in immune regulation: a focus on CCCH zinc finger proteins. Nat. Rev. Immunol. 17, 130-143 (2017).

26. Habacher, C., Ciosk, R. ZC3H12A/MCPIP1/Regnase-1-related endonucleases: an evolutionary perspective on molecular mechanisms and biological functions. Bioessays. 39, 9 (2017).

27. Niu, J. et al. USP10 inhibits genotoxic NF-kB activation by MCPIP1-facilitated deubiquitination of NEMO. EMBO J. 32, 3206-3219 (2013).

28. Oh, Y. T., Qian, G., Deng, J. \& Sun, S. Y. Monocyte chemotactic protein-induced protein-1 enhances DR5 degradation and negatively regulates DR5 activation- 
induced apoptosis through its deubiquitinase function. Oncogene 37, 3415-3425 (2018).

29. $L i, X$. et al. Targeting of tumour-infiltrating macrophages via CCL2/ CCR2 signalling as a therapeutic strategy against hepatocellular carcinoma. Gut 66, 157-167 (2017).

30. Peña, C. G. et al. LKB1 loss promotes endometrial cancer progression via CCL2-dependent macrophage recruitment. J. Clin. Invest. 125, 4063-4076 (2015).

31. Svensson, S. et al. CCL2 and CCL5 are novel therapeutic targets for estrogendependent breast cancer. Clin. Cancer Res. 21, 3794-3805 (2015).

32. Natsagdorj, A. et al. CCL2 induces resistance to the antiproliferative effect of cabazitaxel in prostate cancer cells. Cancer Sci. 110, 279-288 (2019).
33. Kalbasi, A. et al. Tumor-derived CCL2 mediates resistance to radiotherapy in pancreatic ductal adenocarcinoma. Clin. Cancer Res. 23, 137-148 (2017).

34. Chéné, A. L. et al. Pleural effusions from patients with mesothelioma induce recruitment of monocytes and their differentiation into M2 macrophages. J. Thorac. Oncol. 11, 1765-1773 (2016).

35. Kapoor, N. et al. Transcription factors STAT6 and KLF4 implement macrophage polarization via the dual catalytic powers of MCPIP. J. Immunol. 194, 6011-6023 (2015)

36. Brana, I. et al. Carlumab, an anti-C-C chemokine ligand 2 monoclonal antibody, in combination with four chemotherapy regimens for the treatment of patients with solid tumors: an open-label, multicenter phase 1b study. Target Oncol. 10, 111-123 (2015). 